\title{
Análise da fadiga dos músculos paraespinhais em indivíduos saudáveis na posição sentada
}

\author{
Analysis of erector spinae muscle fatigue \\ in healthy subjects in the sitting position
}

Marcio Massao Kawano 1,4 Maryela de Oliveira Menacho 1,4 Beatriz Ito Ramos de Oliveira 2 Marcela Carrilho Boer 2 Roger Burgo de Souza ${ }^{3}$ Jefferson Rosa Cardoso 1,3,4

1 Universidade Estadual de Londrina. Programa de Pós-graduação Associado (Univesidade Estadual de Londrina e Universidade Estadual de Maringá). Londrina, PR. Brasil.

2 Universidade Estadual de Londrina. Curso de Fisioterapia. Londrina, PR. Brasil.

3 Universidade Estadual de Londrina. Departamento de Fisioterapia. Londrina, PR. Brasil.

4 Universidade Estadual de Londrina. Laboratório de Eletromiografia Cinesiológica. Londrina, PR. Brasil.

Recebido em 18/12/07 Aprovado em 24/07/08
Resumo - O objetivo deste estudo foi avaliar os parâmetros de freqüência e amplitude da eletromiografia de superfície durante a fadiga dos músculos paraespinhais em indivíduos saudáveis na posição sentada. Participaram do estudo 11 indivíduos do gênero masculino, saudáveis e livres de sintomas de dor lombar. Um eletromiógrafo de superfície foi utilizado para obtenção dos parâmetros eletromiográficos. Foram posicionados quatro eletrodos lateralmente aos processos espinhosos da primeira e quinta vértebras lombares. Os sinais foram registrados mediante a extensão de tronco na posição sentada contra a resistência de uma célula de carga. Foram utilizadas as cargas de 50\% e 75\% da contração isométrica voluntária máxima, por 25 segundos, para induzir os sujeitos à fadiga muscular. Os sinais eletromiográficos processados pelo algoritmo da Transformada Rápida de Fourier e pela raiz quadrática média foram submetidos à regressão linear para se determinar o índice de fadiga. A freqüência mediana do espectro de potência apresentou valores negativos, assim como a amplitude eletromiográfica valores positivos, o que representa a fadiga muscular. Quando se comparou a altura e o lado, bem como as duas cargas, não foram observadas diferenças estatisticamente significante $(P>0,05)$. Na posição sentada, é possível obter uma melhor fixação da pelve, além de reproduzir uma posição usual das atividades ocupacionais. O protocolo utilizado não causou dor nos sujeitos. Apesar de todos os indivíduos apresentarem fadiga, não houve diferença quanto às regiões da coluna lombar. Sugere-se a utilização da freqüência mediana e da raiz quadrática média concomitante nos estudos de fadiga.

Palavras-chave: Eletromiografia; Análise Espectral; Fadiga; Biomecânica; Região Lombossacral.

Abstract - The aim of this study was to evaluate the frequency and amplitude of surface electromyography during erector spinae fatigue in healthy subjects in the sitting position. Eleven healthy males without low back pain participated in the study. The electromyographic parameters were collected with a surface electromyograph. Four electrodes were positioned lateral to the first and fifth lumbar vertebrae. The electromyographic signals were recorded during isometric trunk extension against a load cell in the sitting position. Loads of $50 \%$ and $75 \%$ of maximal voluntary isometric contraction were applied for 25 seconds to induce muscle fatigue. The signals processed using a Fast Fourier Transform algorithm and root mean square were submitted to linear regression to determine the fatigue index. The median frequency of the power spectrum presented negative values and the root mean square presented positive values, indicating muscle fatigue. No significant differences were observed when comparing level and side, or between loads ( $P$ $>0.05$ ). The sitting position provides a better pelvis fixation and reproduces the usual position of occupational activities. The protocol used did not cause pain. Although all subjects presented erector spinae fatigue, no differences were observed between lumbar regions. The concomitant use of median frequency and root mean square in fatigue studies is suggested.

Key words: Electromyography; Spectrum Analysis; Muscle Fatigue; Biomechanics; Lumbosacral Region. 


\section{INTRODUÇÃO}

A função dos músculos paraespinhais tem sido amplamente estudada uma vez que estes são responsáveis por iniciar e controlar todos os movimentos da coluna vertebral ${ }^{1}$. Além disso, realizam a estabilização extrínseca da coluna lombar e, portanto, protegem os elementos espinhais não contráteis de estresses potencialmente danosos ${ }^{2,3}$. O recrutamento desse grupo muscular é constantemente requisitado durante longos períodos de trabalho e atividades da vida diária, geralmente, realizadas na posição sentada.

A fadiga muscular é definida como falha ou diminuição da força durante atividades de sustentação ${ }^{4}$. Quando ocorre fadiga nos paraespinhais, há um controle diminuído dos movimentos de tronco e alteração na coordenação das atividades dos mesmos ${ }^{1}$. Deficiências no controle neuromuscular desses músculos levam à perda de estabilidade na coluna que os expõem a estresses, tensões repetitivas e maior sobrecarga na coluna ${ }^{5,6}$. Conseqüentemente, há predisposição à instalação e recorrência de dor, como por exemplo, a lombalgia ${ }^{3}$.

A eletromiografia de superfície (EMG) é amplamente utilizada para a avaliação da fadiga muscular ${ }^{4,5}$. Por meio desta ferramenta, é possível obter os sinais eletromiográficos que possibilitam verificar a ocorrência da mesma. Para esta análise, durante contrações isométricas sustentadas, observa-se um aumento da amplitude dos sinais eletromiográficos e uma compressão do espectro para baixas freqüências que indicam a ocorrência de fadiga ${ }^{2,7,8}$. Por meio da análise espectral dos sinais eletromiográficos, é possível obter a freqüência mediana (FM). Esta é definida como a freqüência que divide o espectro de potência em duas regiões simétri$\mathrm{cas}^{4}$. Este parâmetro eletromiográfico apresenta maior confiabilidade ${ }^{9}$ e são menos sensíveis aos ruídos de alta freqüência ${ }^{10}$ quando comparados a outras variáveis de medida do espectro de potência, como por exemplo, a freqüência média. Embora estudos prévios utilizem apenas a análise espectral para detectar a fadiga, para Öberg 8 , é necessária a utilização deste parâmetro em conjunto com a amplitude eletromiográfica para a identificação da fadiga.

Há na literatura estudos prévios que envolveram a EMG e a análise da resistência dos paraespinhais e que classificaram os indivíduos com e sem lombalgia, treinados e não treinados e até mesmo durante a reabilitação ${ }^{11-14}$. Entretanto, a maioria destes estudos foi realizada com os indivíduos na posição ortostática ou em prono. Sabe-se que durante a extensão de tronco, muitos outros músculos estão envolvidos ${ }^{15}$ e qualquer falta de estabilização da pelve ou outra estrutura, pode acarretar em compensações posturais que afetam significativamente os resultados dos testes. Acredita-se que na posição sentada, há uma melhor fixação da pelve e maior estabilidade. Além disso, essa posição reproduz uma situação comum do cotidiano o que a torna preferencial para a perspectiva clínica e de reabilitação ${ }^{16}$. Por outro lado, uma melhor compreensão do comportamento da fadiga na posição sentada, pode contribuir para a prevenção da dor lombar. Como hipótese para o estudo, acredita-se que esta posição seja mais confortável e não cause dor para o indivíduo, além de demonstrar a fadiga pelos dois parâmetros eletromiográficos. Dessa forma, o objetivo deste estudo foi avaliar os parâmetros de freqüência e amplitude da eletromiografia de superfície, durante a fadiga dos músculos paraespinhais, em indivíduos saudáveis na posição sentada.

\section{PROCEDIMENTOS METODOLÓGICOS}

\section{Sujeitos}

Participaram deste estudo 11 indivíduos do gênero masculino, recrutados por conveniência, com idades entre 20 a 40 anos $(\bar{x}=31,4$ anos, $\mathrm{DP}=8,2)$; massa corpórea $\bar{x}=69,2 \mathrm{~kg}$, DP $=$ 5,3; estatura $\bar{x}=175,1 \mathrm{~cm}, \mathrm{DP}=6,1 \mathrm{e}$ índice de massa corpórea $\bar{x}=24,6 \mathrm{~kg} / \mathrm{m}^{2}, \mathrm{DP}=2,1$. Como critério de inclusão, todos os sujeitos deveriam ser saudáveis, ter entre 20 a 40 anos e serem do gênero masculino. Como critério de exclusão, indivíduos com alteração postural que comprometesse o funcionamento adequado dos músculos extensores do tronco, lesões osteomioarticulares nos últimos 6 meses, história de cirurgia em membros ou coluna e não ter histórico de episódio de dor lombar nos últimos 6 meses. Todo o protocolo de pesquisa foi aprovado pelo Comitê de Ética em Pesquisada Universidade Estadual de Londrina (CEP 039/04) e todos os participantes foram informados e assinaram o termo de consentimento livre e esclarecido.

\section{Equipamentos}

Para obtenção dos dados, foi utilizado um eletromiógrafo de superfície com dezesseis 
canais (EMG System do Brasil), composto por um conversor A-D (analógico-digital) de doze bits de resolução. Cada canal é acoplado a dois eletrodos ativos e um de referência. Os eletrodos circulares de prata/cloreto de prata $(\mathrm{Ag} / \mathrm{AgCl})$ (formato oval $43 \times 45 \mathrm{~mm}$ ) foram conectados a um pré-amplificador de alta impedância (1.0 x $10^{12} \mathrm{Ohm}$ ), com ganho de $20 \mathrm{x}$, razão de rejeição do modo comum maior que $100 \mathrm{~dB}$. Os sinais foram ajustados para 1000 amostras por segundo e o filtro numa freqüência de passagem de 20 a $450 \mathrm{~Hz}$.

Uma célula de carga (Alfa Instrumentos; tração/compressão - Modelo SV - $200 \mathrm{~kg}$ ) foi fixada em um colete e a outra extremidade à parede para quantificar a contração isométrica voluntária máxima (CIVM) e dos testes. Para o posicionamento sentado dos indivíduos durante os testes, utilizou-se uma cadeira extensora. Além disso, para o posicionamento adequado da articulação do quadril em $30^{\circ}$ de flexão, utilizou-se um goniômetro universal.

\section{Procedimentos}

Os voluntários permaneceram sentados enquanto os músculos foram localizados por meio da palpação, precedido por tricotomia e limpeza do local com álcool 70\% para diminuir a impedância da pele. Os eletrodos ativos de prata-cloreto de prata $(\mathrm{Ag} / \mathrm{AgCl})$ foram colocados sobre a região dos músculos paraespinhais na altura da primeira (L1D e L1E) e da quinta vértebras lombares (L5D e L5E) do lado direito e esquerdo, sobre os músculos longuíssimo do dorso e multífidos, respectivamente ${ }^{16}$. Reconhecem-se as dificuldades na avaliação do multífidos, portanto, considerou-se uma maior sensibilidade na detecção da atividade do longuíssimo do dorso adjacente do que a própria atividade dos multifí$\operatorname{dos}^{17}$. A distância centro-a-centro dos eletrodos foi de dois centímetros paralelos às fibras musculares e do centro dos eletrodos até o centro do processo espinhoso foi de três centímetros ${ }^{18}$. O eletrodo de referência foi colocado no punho direito. Todo o procedimento de colocação dos eletrodos seguiu as recomendações do SENIAM (Surface-EMG for the Non Invasive Assessment of Muscle $)^{19}$. Todos os participantes assinalaram a escala visual análoga (EVA) antes e após todo o procedimento para assegurar que o protocolo não causaria dor nos indivíduos.

A posição dos testes de fadiga e para a obtenção da CIVM foi a mesma (Figura 1). Cada participante foi colocado na posição sentada em uma cadeira extensora com a pelve devidamente fixada por cinto, com um colete ajustável acoplado à célula de carga. Para determinar a CIVM, o participante, a $30^{\circ}$ de flexão de quadril, realizou três tentativas com a extensão do tronco contra a resistência da célula de carga por cinco segundos de contração e dez segundos de repouso. Das três tentativas, a maior foi utilizada para a normalização dos dados. Durante o teste de CIVM, os participantes receberam instruções verbais e motivação.

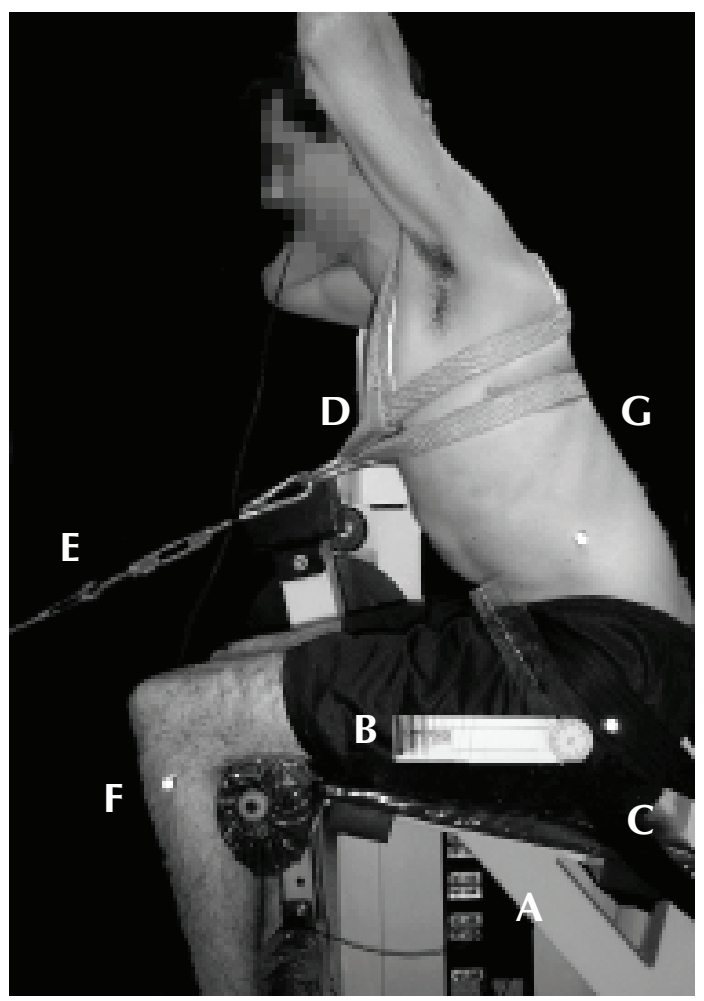

Figura 1. Postura e equipamentos para os testes de contração isométrica voluntária máxima e de fadiga muscular. Cadeira de teste (A); goniômetro (B); cintos (C); colete (D); cabo acoplado à célula de carga (E); marcadores reflexivos (F); eletrodos de superfície (G).

Para determinar a fadiga muscular, dois testes foram realizados: um submetido a $50 \%$ e o outro a $75 \%$ da CIVM. Por meio de sorteio simples, determinou-se a ordem da realização dos testes. $O$ protocolo consistia na extensão de tronco nestas duas cargas, com duração de 25 segundos em cada teste. Com o participante devidamente posicionado, o primeiro teste foi realizado com a execução do movimento de extensão do tronco. Para estes procedimentos, um monitor foi posicionado a frente dos participantes para um feedback visual da carga a ser exercida. O indivíduo realizava o movimento de extensão de tronco de modo que ao tracionar a 
célula de carga, ele deveria verificar no monitor a carga, previamente informada, que representava 50 ou $75 \%$ da CIVM. Entre um teste e outro, foi estipulado cinco minutos de repouso. Adotou-se como critério para o encerramento do teste e conseqüentemente, a exclusão do indivíduo do estudo, a incapacidade de manter em no máximo $10 \%$ abaixo da carga teste.

\section{Análise dos sinais eletromiográficos}

Após as coletas, os dados foram analisados pelo programa de aquisição de dados (AqDados, 5.0) e no programa AcqKnowledge 3.7.1. Dois parâmetros eletromiográficos de fadiga foram verificados: no domínio da freqüência, a FM, ao longo do tempo e no domínio temporal, a amplitude eletromiográfica expresso na RMS (root mean square) - raiz quadrática média do quadrado da grandeza. Para determinar a FM, os dados foram processados pelo algoritmo da Transformada Rápida de Fourier. A análise eletromiográfica da fadiga foi realizada por meio da FM e da RMS, calculado para cada 2,5 segundos de intervalo registrado. Utilizou-se a regressão linear simples para determinar a taxa de mudança da FM e da RMS em função do tempo $(t=0$ até $t=25 \mathrm{~s})$. Para isto, obteve-se o slope (coeficiente angular da reta) de cada um dos quatro músculos nas diferentes cargas. Os valores da RMS foram normalizados com as correspondentes CIVM ${ }^{20,21}$ e quando os slopes (\%/s) apresentaram-se positivos, interpretou-se como fadiga. Já os slopes da FM ( $\mathrm{Hz} / \mathrm{s})$ quando negativos, evidenciam a ocorrência de fadiga muscular.

\section{Análise Estatística}

Os dados antropométricos foram avaliados quanto à distribuição de normalidade e são apresentados de em forma de média $(\bar{x})$ e desvio-padrão (DP) por assumirem os pressupostos necessários. Para a comparação das médias dos slopes da FM e RMS dos músculos paraespinhais de L1 e L5, de ambos os lados, foi utilizada a Análise de Variância (ANOVA) de medidas repetidas. O local do músculo e o lado foram considerados como fatores "intra" (within-subject) indivíduo. Foi aplicado o teste de esfericidade de Mauchly W e, quando este foi violado, correções técnicas foram realizadas utilizando-se o teste de Huynh-Feldt. Caso o teste $\mathrm{F}$ fosse significante, complementar-se-ia a análise pelo teste de comparações de Bonferroni. A significância estatística $(\alpha)$ foi estipulada em 5\%. Para todas as análises, foi utilizado o programa SPSS (Statistical Package for Social Sciences, versão 13.0).

\section{RESULTADOS}

Todos os 11 sujeitos conseguiram encerrar os testes de 25 segundos nas duas cargas e não relataram dor nem antes e nem após os testes de fadiga.

Os sujeitos no teste a $50 \%$ da CIVM obtiveram valores médios da FM inicial e final, respectivamente, em Hertz $(\mathrm{Hz}): \mathrm{L} 1 \mathrm{D}=92,6$ e 89,5; L1E = 96,8 e 91,4; L5D = 111,2 e 107,1; $\mathrm{L} 5 \mathrm{E}=119,1$ e 120,5. As médias da FM a 75\% da CIVM inicial e final, respectivamente foram em Hz: L1D = 86,7 e 82,5; L1E = 90,1 e 88,2; L5D $=107,2$ e 100,$1 ; \mathrm{L} 5 \mathrm{E}=111,9$ e 109,8 .

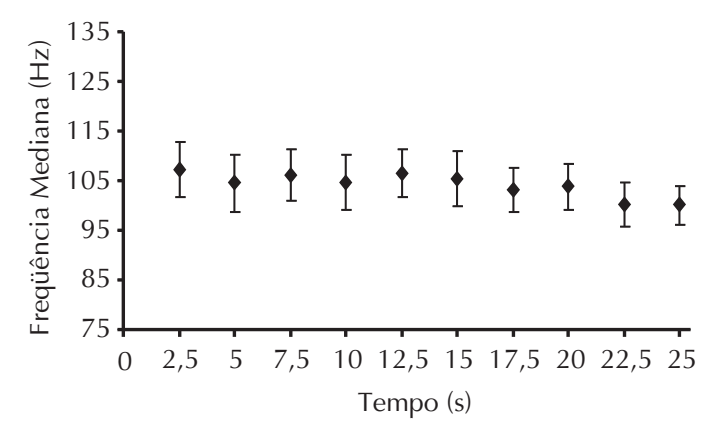

Figura 2. Média e erro padrão da média das FM em L5D à 75\% da CIVM dos 11 indivíduos.

Todos os valores de slopes da reta de regressão da FM foram negativos o que representa o declínio da FM ao longo do tempo. Entretanto, não foi observada diferença estatisticamente significante quando se comparou os lados e os segmentos avaliados $(P>0,05)$. A Figura 2 representa a média e erro padrão da FM em L5D à 75\% dos 11 indivíduos. Os outros músculos apresentaram mesmo comportamento da freqüência mediana ao longo do teste de fadiga.

Os slopes que representam as RMS obtiveram valores positivos, com exceção de um sujeito que apresentou valor negativo em L5E quando submetido a 50\% da CIVM. Apesar dos valores a $75 \%$ da CIVM serem maiores do que a 50\%, não houve diferença estatisticamente significante $(P>0,05)$ assim como, quando comparados os segmentos e os lados. A Figura 3 representa média e erro padrão da RMS L5D a 75\% da CIVM de toda a amostra.

$\mathrm{Na}$ Tabela 1, constam as médias dos slopes da FM e da RMS nos testes de fadiga a 50 e $75 \%$ da CIVM. 


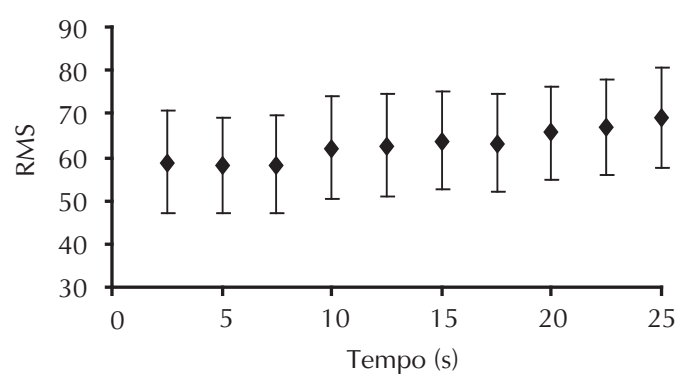

Figura 3. Média e erro padrão da média da RMS em L5D a $75 \%$ da CIVM dos 11 indivíduos.

Tabela 1. Média dos slopes da freqüência mediana (FM) e da raiz quadrada da média (RMS) a $50 \%$ e $75 \%$ da CIVM dos 11 indivíduos.

\begin{tabular}{lcc}
\hline $\begin{array}{l}\text { Segmento } \\
\text { vertebral }\end{array}$ & $\begin{array}{c}\mathrm{FM}(\mathrm{Hz} / \mathrm{s}) \\
\bar{x}(\mathrm{EP})\end{array}$ & $\begin{array}{c}\mathrm{RMS}(\% / \mathrm{s}) \\
\bar{x}(\mathrm{EP})\end{array}$ \\
\hline L1D 50\% & $-0,37(0,18)$ & $0,04(0,12)$ \\
L1E 50\% & $-0,87(0,27)$ & $0,06(0,12)$ \\
L5D 50\% & $-0,55(0,14)$ & $0,03(0,10)$ \\
L5E 50\% & $-0,56(0,17)$ & $-0,01(0,14)$ \\
L1D 75\% & $-0,37(0,20)$ & $0,29(0,25)$ \\
L1E 75\% & $-0,46(0,11)$ & $0,25(0,23)$ \\
L5D 75\% & $-0,65(0,31)$ & $0,43(0,22)$ \\
L5E 75\% & $-0,39(0,32)$ & $0,40(0,22)$ \\
\hline
\end{tabular}

$\bar{x}$ = média; $\mathrm{EP}=$ erro padrão da média; $\mathrm{L} 1$ = primeira vértebra lombar; $\mathrm{L} 5$ = quinta vértebra lombar; $\mathrm{D}=$ lado direito; $\mathrm{E}=$ lado esquerdo.

\section{DISCUSSÃO}

Estudos prévios que envolveram a análise da fadiga dos paraespinhais utilizaram como parâmetro eletromiográfico apenas a análise espectral $^{9-12}$. Entretanto, para Öberg ${ }^{8}$ somente o uso de um parâmetro, análise espectral ou da amplitude eletromiográfica, não fornece informação suficiente a respeito do processo de fadiga muscular. Há uma significante influência da fadiga sobre estes dois parâmetros. A amplitude EMG pode se elevar devido ao aumento de cargas ou devido à fadiga muscular, mas isso não pode ser diferenciado. Conseqüentemente, há a necessidade de algum outro indicador de fadiga muscular. A análise espectral é considerada como mais específica para avaliar a fadiga muscular. Por esta razão, o parâmetro espectral deve ser avaliado junto com a amplitude.

$\mathrm{O}$ aumento na amplitude eletromiográfica (RMS) apresentado pela maioria dos sujeitos neste estudo, reflete num recrutamento adicional de unidades motoras devido à fadiga das fibras musculares ${ }^{20,21}$. Além disso, este aumento pode ser atribuído, também, a uma maior fre- qüência de despolarizações da fibra muscular ${ }^{22}$, ou até mesmo em conseqüência de um aumento na carga imposta ao músculo ${ }^{8}$. Um dos sujeitos apresentou, inicialmente, um aumento da RMS, seguido posteriormente de um decréscimo. Este fenômeno pode ser explicado pelo desrecrutamento de fibras musculares devido ao excesso de fadiga ${ }^{23,24}$. De acordo com Clark et al. e Barbosa et al. este decréscimo da RMS, após um aumento, ocorre devido à redução na taxa de despolarização das fibras musculares ${ }^{23,24}$. Desta forma, para manter o torque necessário, outros músculos, os sinergistas, agem como substitutos daquele que se encontra fatigado. Este fenômeno de desrecrutamento de fibras musculares ocorre com o objetivo de prevenir lesões no sistema músculo-esquelético ${ }^{24}$.

Os extensores de coluna apresentam uma alta resistência à fadiga em comparação com outros grupos musculares. Isto é atribuído à sua composição muscular, capilaridade favorável e a uma possível distribuição da carga com outros músculos sinergistas ${ }^{21}$. Estudos em músculos paraespinhais de sujeitos assintomáticos sugerem que declínios na FM induzidas pela fadiga estão relacionadas com a quantidade de fibras tipo $\mathrm{I}^{1}$. Quanto maior a contingência de fibras tipo I, maior é a resistência do músculo à fadiga, mais lenta é queda da FM e (geralmente) o período de sustentação da contração é prolongado1. A área correspondente aos músculos ocupados por fibras tipo II foi relacionada com o aumento no declínio da FM dos músculos da coluna. Portanto, a ausência de um aumento no declínio da FM, neste estudo, sugere ser devido a um número menor de fibras tipo II, como descrito em um estudo prévio ${ }^{8}$.

Quando se comparou a fadiga nas duas cargas, 50\% e 75\% da CIVM, não se constataram diferença estatisticamente significante. $\mathrm{O}$ mesmo ocorreu quando se comparou a fadiga dos músculos na altura de L1 e L5 para FM e RMS. Isto contraria estudos prévios que relataram maiores índices de fadiga em L $5^{11,12,21}$. Estes autores atribuem às regiões mais baixas da coluna lombar, a responsabilidade de sustentar maiores cargas. Segundo Peach et al. ${ }^{25}$ existem mais fibras do tipo I em regiões acima de L5, o que justificaria maior fadiga na altura de L1. Entretanto, de acordo Kankaanpää et al. ${ }^{10}$, na posição sentada, há uma maior fadiga dos músculos glúteo máximo em relação à região de L5. Apesar de estar em posição desfavorável para 
a geração de torque, este músculo se contrai e auxilia na estabilização do individuo na posição sentada. Estas transferências de sobrecargas ocorrem devido à ligação da coluna lombar com os músculos extensores de quadril pela fáscia toracolombar. Assim, durante exercícios fatigantes, os extensores de quadril recebem sobrecargas provenientes da região lombar.

No presente estudo, utilizou-se um protocolo de avaliação na posição sentada, ao invés de prono ou ortostatismo como visto em estudos prévios. Geralmente, as atividades ocupacionais são exercidas grande parte do dia na posição sentada. Segundo Cassisi et al. ${ }^{26}$, a extensão isométrica na posição sentada é mais eficiente do que na posição ortostática. Para Allison e Henry $^{15}$, os sujeitos podem realizar o uso de estratégias de compensação para executar o movimento desejado na posição ortostática. Portanto, na posição sentada é possível obter uma melhor fixação da pelve e o indivíduo sente-se mais confortável para executar a extensão de tronco. Além disso, esta posição reproduz uma situação comum do cotidiano.

Uma limitação do estudo foi a não realização da medida da atividade eletromiográfica de outros músculos, que não atuam como motor primário da extensão de tronco, mas como sinergistas do movimento ou como acessórios. Estas mensurações poderiam, em parte, trazer informações adicionais a respeito da atuação de outros músculos neste movimento, como o glúteo máximo, reto abdominal, oblíquos externos e até mesmo de segmentos da coluna torácica e cervical.

\section{CONCLUSÃO}

No presente estudo, verificou-se a aplicabilidade dos dois parâmetros eletromiográficos na análise da fadiga muscular. A FM indicou valores negativos de slopes, o que representa a fadiga muscular. Por outro lado, os valores da RMS foram positivos o que indica um aumento do recrutamento de unidades motoras para manter a força requerida. Os indivíduos ao realizar o teste na posição sentada não apresentaram dor. Sugere-se que novos estudos utilizem o protocolo na posição sentada, além dos dois parâmetros FM e RMS concomitante.

\section{REFERÊNCIAS BIBLIOGRÁFICAS}

1. Mannion AF. Fiber type characteristics and function of the human paraspinal muscles: normal values and changes in association with low back pain. J Electromyogr Kinesiol 1999;9(6):363-77.

2. Crossman K, Mahon M, Watson PJ, Oldaham JA, Cooper RG. Chronic low back pain-associated paraspinal muscle dysfunction is not the result of a constitutionally determined "adverse" fiber type composition. Spine 2004;29(6):628-34.

3. Cholewicki J, Van Nliet IV JJ. Relative contribution of trunk muscles to the stability of the lumbar spine during isometric exertions. Clin Biomech 2002;17(2):99-105.

4. Dimitrova NA, Dimitrov GV. Interpretation of EMG changes with fatigue: facts, pitfalls, and fallacies. J Electromyogr Kinesiol 2003;13(1):13-36.

5. Van Dieen JH, Selen LP, Cholewicki, J. Trunk muscular activation in low-back pain patients, an analysis of literature. J Electromyogr Kinesiol 2003;13(4):333-51.

6. O'Sullivan P. Diagnosis and classification of chronic low back pain disorders: maladaptive movement and motor control impairments as underlying mechanism. Man Ther 2005;10(4):242-55.

7. Cram JR. The history of electromyography. Appl Psychophysiol Biofeedback 2003;28(2):81-91.

8. Öberg T. Muscle fatigue and calibration of EMG measurements. J Electromyogr Kinesiol 1995;5(4):239-43.

9. Kramer M, Ebert V, Kinzl L, Dehner C, Elbel M, Hartwig E. Surface electromyography of the paravertebral muscles in patients with chronic low back pain. Arch Phys Med Rehabil 2005;86(1):31-6.

10. Kankaanpää M, Taimela S, Laaksonen D, Hänninen $\mathrm{O}$, Airasksinen O. Back and hip extensor fatigability in chronic low back pain patients and controls. Arch Phys Med Rehabil 1998;79(4):412-7.

11. Farina D, Gazzoni M, Merletti R. Assessment of low back muscle fatigue by surface EMG signal analysis: methodological aspects. J Electromyogr Kinesiol 2003;13(4):319-32.

12. Roy SH, De Luca CJ, Casavant DA. Lumbar muscle fatigue and chronic lower back pain. Spine 1989;14(9):992-1001.

13. Umezu Y, Kawazu T, Takima F, Ogata H. Spectral electromyographic fatigue analysis of back muscles in healthy adult women compared with men. Arch Phys Med Rehabil 1998;79(5):536-38.

14. Sung PS. Multifidi muscles median frequency before and after spinal stabilization exercises. Arch Phys Med Rehabil 2003;84(9):1313-8.

15. Allison GT, Henry SM. Trunk muscle fatigue during a back extension task in standing. Man Ther 2001;6(4):221-28.

16. Elfving B, Dedering A. Task dependency in back muscle fatigue - Correlations between two test methods. Clin Biomech 200;22(1):28-33.

17. Stokes IA, Henry SM, Single RM. Surface EMG electrodes do not accurately record from lumbar multifidus muscles. Clin Biomech 2003;18(1):9-13.

18. Dedering A, Oddsson L, Harms-Ringdahl K, Né- 
meth G. Electromyography and ratings of lumbar muscle fatigue using a four-level staircase protocol. Clin Biomech 2002;17(1):171-76.

19. Hermens HJ, Freriks B, Disselhorst-Klug C, Rau G. Development of recommendations for SEMG sensors and sensor placement procedures. J Electromyogr Kinesiol 2000;10(5):361-74.

20. Silva Jr RA, Arsenault B, Gravel D, Larivière C, Oliveira Jr E. Back muscle strength and fatigue in healthy and chronic low back pain subjects: a comparative study of 3 assessment protocols. Arch Phys Med Rehabil 2005;86(4):722-9.

21. Larivière C, Gravel D, Gagnon D, Gardiner P, Arsenault $A B$, Gaudreault N. Gender influence on fatigability of back muscles during intermittent isometric contractions: A study of neuromuscular activation patterns. Clin Biomech 2006;21(9):893-904.

22. Ng JKF, Richardson CA, Jull GA. Electromyographic amplitude and frequency changes in the iliocostalis lumborum and multifidus muscles during a trunk holding test. Phys Ther 1997;77(9):954-61.

23. Clark BC, Manini TM, Ploutz-Snyder LL. Derecrutiment of the lumbar musculature with fatiguing trunk extension exercise. Spine 2003;28(3):282-7.

24. Barbosa FSS, Gonçalves M. Comparação entre protocolos de exaustão e de 30 segundos utilizados na avaliação da fadiga eletromiográfica dos músculos eretores da espinha. Rev Bras Fisioter 2005;9(1):77-83.
25. Peach JP, McGill S. Classification pf low back pain with the use of spectral electromyogram parameters. Spine 1998;23(10):1117-23.

26. Cassisi JE, Robinson ME, O'Conner P, MacMillan M. Trunk strength and lumbar paraspinal muscle activity during isometric exercise in chronic low-back pain patients and controls. Spine 1993;18(2):245-51.

\section{Agradecimento}

Ao Fisioterapeuta Alexandre Henrique Nowotny, Mestre em Ciências da Saúde-UEL, pelo empréstimo da célula de carga utilizada neste estudo. Ao CNPq (Conselho Nacional de Desenvolvimento Científico e Tecnológico) pelo apoio por meio da Bolsa Produtividade em Pesquisa ao último autor.

\footnotetext{
Endereço para correspondência

Jefferson Rosa Cardoso

Hospital Universitário Regional Norte do Paraná Departamento de Fisioterapia Avenida Robert Kock, nº 60 86038-440, Londrina, PR. Brasil

E-mail: jeffcar@uel.br
} 